\title{
Research Paper \\ The Effectiveness of Acceptance and Commitment Therapy on Chronic Fatigue Syndrome and Pain Perception in People With Multiple Sclerosis
}

\author{
Maryam Davoodi ${ }^{1}$ (), * Leila Shameli ${ }^{1}$ (), Habib Hadianfard ${ }^{2}$ (c)
}

1. Department of Psychology, Faculty of Letters and Humanities, Salman Farsi University of Kazerun, Kazerun, Iran

2. Department of Clinical Psychology, Faculty of Education \& Psychology, Shiraz University, Shiraz, Iran.

\begin{tabular}{|c|c|}
\hline $\begin{array}{l}\text { Use your device to scan } \\
\text { and read the article online }\end{array}$ & dtation Davoodi M, Shameli L, Hadianfard H. The Effectiveness of Acceptance and Commitment Therapy on Chronic Fa- \\
\hline 回倌神回 & tigue Syndrome and Pain Perception in People With Multiple Sclerosis. Iranian Journal of Psychiatry and Clinical Psychology. \\
\hline prtar & 2019; 25(3):250-265. http://dx.doi.org/10.32598/ijpcp.25.3.250 \\
\hline 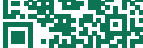 & doi http://dx.doi.org/10.32598/ijpcp.25.3.250 \\
\hline
\end{tabular}

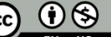

Received: 14 Nov 2018 Accepted: 01 Jun 2019 Available Online: 01 Oct 2019

Key words:

Acceptance and commitment therapy, Chronic fatigue syndrome, Pain perception, Multiple sclerosis

\begin{abstract}
A B S T RACT
Objectives The present study investigated the effectiveness of Acceptance and Commitment Therapy (ACT) on Chronic Fatigue Syndrome (CFS) and Pain Perception (PP) in people with Multiple Sclerosis (MS). Methods This was a quasi-experimental study with a pre-test, post-test and a control group design. The statistical population was all individuals with MS referring to the MS Society of Ahvaz, Iran, in 2018. Thirty patients with SFS were selected and randomly assigned into two groups of test and control (15 per group). Moreover, Multidimensional Fatigue Inventory (MFI) (was used to measure chronic fatigue symptoms) and Fatigue Severity Scale (FSS) and McGill Pain Questionnaire (MGPQ) were used for data collection. The achieved data were analyzed by Multivariate Analysis of Covariance (MANCOVA) in SPSS. Results The MANCOVA results revealed a significant difference between two groups in the following variables: perception of sensory pain $(F=14.70, P \leq 0.001)$, perception of pain assessment $(F=70.50, P \leq 0.01)$, perception of various pain ( $F=8.13, P \leq 0.001), P P(F=14.68, P \leq 0.001$,) and $C F S(F=4, P \leq 0.05)$.

Conclusion The study finding suggested that ACT was effective in reducing the severity of CFS and PP in the experimental group; this reduction has led to a relative improvement in MS condition. Therefore, clinicians working in health centers can use this treatment along with pharmacotherapy.
\end{abstract}

\section{Extended Abstract}

\section{Introduction}

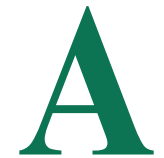

Breakdown in the myelin's neuronal brain cells in the central nervous system leads to generating Multiple Sclerosis (MS). The prevalence of MS in Iran is 40 per 100,000 people [1]. Recent research has identified environmental factors, such as stress, genetics, as well as immunological and microbiological matters, as the causes of this disease [4]. MS is categorized as an autoimmune disease, where the immune system attacks the brain and spinal cord and disrupts their functions [5].

Studies have reported that psychological distress in these individuals is associated with biological changes [7]. A high rate of psychological problems has been reported in patients with MS $[8,9]$, which patients consider them as the cause of their pain [10].

Pain is an unpleasant feeling transmitted by the sensory neurons to the brain. However, pain falls beyond physical sensations or consciousness; Pain Perception (PP) also includes a mental interpretation of discomfort [12]. There-

* Corresponding Author:

Leila Shameli, PhD.

Address: Department of Psychology, Faculty of letters and humanities, Salman Farsi University of Kazerun, Kazerun, Iran.

Tel: +98 (933) 6598441

E-mail: Ishameli29@yahoo.com 
fore, PP involves the objective experience of physical processes and a mental experience, including affective-emotional characteristics [13]. Furthermore, distress, disease perception, fatigue, and cognitive-behavioral factors are associated with pain intensity and PP [14].

Another common symptom that is commonly reported in MS patients is CFS. It is known as a specific problem, and decreases the quality of life of patients; however, the exact mechanism of it is unclear [15]. CFS is commonly associated with mild disease and may be the first symptom [16]. MS Council of Clinical Practice (1998) defined MS fatigue as "the lack of physical and psychological energy; i.e. experienced by the individuals or their caregivers and affects the individuals' daily living activities" [20]. The study of MS, chronic fatigue, and PP suggests this disease is associated with many psychological problems for the patient.

Therefore, studying the effectiveness of psychological interventions on the important mental variables of these patients is necessary. One of the beneficial psychological therapies in this regard can be Acceptance and Commitment Therapy (ACT) [26]. ACT is a mindfulness-based behavioral therapy. Reducing disease signs is not the purpose of ACT. In this therapy, the goal is to create a rich and meaningful life, while the patient accepts the inevitable suffering.

CFS and chronic pain are among the main causes of MS complications with cognitive and psychological dimensions. MS has not been treated conclusively, and most of the existing treatments only reduce symptoms or slow down the disease progression [1]. However, there is evidence of the usefulness of Cognitive Behavioral Therapy (CBT) in treating depression to cope with MS [29]. ACT is a modern approach, compared to CBT; i.e. used to improve one's quality of life and ability to stay active and acting in accordance with their values [30]. Therefore, the present study aimed to explore the effectiveness of ACT on CFS and $\mathrm{PP}$ in people with MS.

\section{Methods}

Thus, this was an applied and quasi-experimental research with a pre-test, post-test design. The statistical population includes all people with MS referring to the MS Society of Ahvaz. Thirty of these patients who were diagnosed with CFS by multidimensional fatigue inventory and whose condition was classified by a physician's diagnosis according to the Expanded Disability Status Scale was poorly classified were selected as study samples.

\section{Results}

Multivariable Analyze of Covariance (MANCOVA) was used to analyze the obtained data. Based on MANCOVA for PP and CFS scores, the results obtained from the Wilks Lambda test were at $\% 95$ confidence level $(\mathrm{F}=0.77$, $\mathrm{P}<0.02$ ). Thus, the two groups significantly differed in terms of at least one of the dependent variables (Table 1).

Wilks Lambda's test results of PP were significant for the subscales of sensory of pain perception, emotional perception of pain, and various pain perception $(\mathrm{P} \leq 0.05, \mathrm{~F}=5.70)$ (Table 2). The difference between the two groups in the sensory of pain perception ( $\mathrm{F}=14.17, \mathrm{P}<0.001)$, assessment of pain perception $(\mathrm{F}=10.54, \mathrm{P} \leq 0.003)$, and various perceptions of pain $(\mathrm{F}=105.224, \mathrm{P} \leq 0 / 05)$ was significant.

Table 1. MANCOVA results for the mean pre-test and post-test scores and controls

\begin{tabular}{cccccc}
\hline Variables & Sum of Squares & df & F & P & Eta \\
\hline Pain perception & 108.72 & 1 & 15.15 & 0.001 & 0.96 \\
Chronic fatigue syndrome & 266.37 & 1 & 4.27 & 0.04 & 0.51 \\
\hline
\end{tabular}

Iranian Journal of
PSYCHIATRY AND CUINICAL PSYCHOLOGY

Table 2. MANCOVA results for the mean pre-test and post-test scores and controls

\begin{tabular}{cccccc}
\hline Variables & Sum of Squares & df & F & P & Eta \\
\hline Sensory of pain perception & 455.02 & 1 & 17.14 & 0.001 & 0.89 \\
Emotional pain perception & 0 & 1 & 0 & 0 & 0 \\
Assessment of pain perception & 9.50 & 1 & 10.54 & 0.003 & 0.88 \\
Various pain perception & 105.24 & 1 & 9.51 & 0.05 & 084 \\
\hline
\end{tabular}




\section{Discussion}

The study results indicated that $\mathrm{ACT}$ could reduce fatigue, and PP in patients with MS. ACT helps patients to experience their inner experience as a thought, and instead of responding to them, follow their values in life and things that matter to them.

According to ACT, CFS, and PP in MS patients are due to their beliefs that their fatigue and pain are directly related to their illness. Furthermore, because they are unable to control their fatigue and pain, they try to avoid further activities. As a result, the patients fall in a defective loop and strengthen fatigue and distress. The current treatment, by breaking this faulty cycle, attempted to reduce fatigue and pain in MS patients.

\section{Ethical Considerations}

\section{Compliance with ethical guidelines}

In this research, the form of ethical considerations was filled by the patients and was followed by the researchers. Also, the participants signed the informed consent form and had the right to leave the study at any time.

\section{Funding}

The present research was extracted from a master thesis approved by Maryam Davoodi in the Faculty of Letters and Humanities, Department of General Psychology at Salman Farsi University of Kazerun.

\section{Authors contributions}

Conceptualization, validation and project management: All authors; Methodology: Maryam Davoodi; Analysis: Leila Shameli; Research: Maryam Davoodi; Sources: Maryam Davoodi; Writing draft: Maryam Davoodi; Editing and finalizing: Leila Shameli.

\section{Conflicts of interest}

The authors declared no conflicts of interest.

\section{Acknowledgements}

We would like to thank the personnel of the MS Association in Ahvaz city especially Dr. Majdi Nasab and Dr. Nedamat and all patients participated in this study. 


\title{
تأثير دومان مبتنى بر يذيرش و تعهد بر نشانكًان خستكًى مزمن و ادراك درد افراد مبثلا به مالتيّل اسكولوروزيس دومان ميتي
}

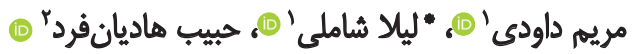 \\ ا- آتروه روانشناسى، دانشكده ادبيات و علوم أسانى، دانشعاه سلمان فارسى كازرون، كازرون، ايران.

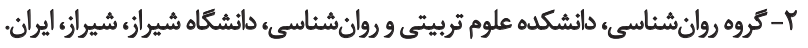

\begin{abstract}
حكبد

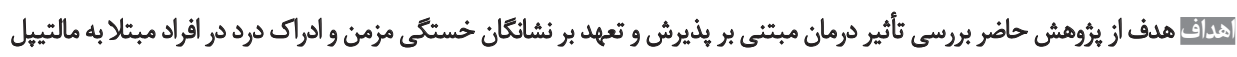
اسكولوروزيس بود.

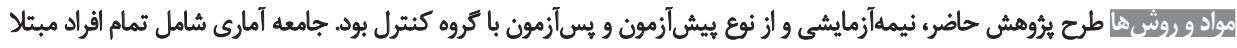

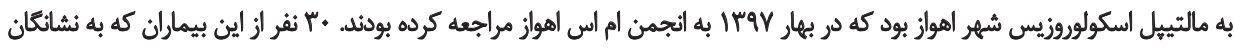

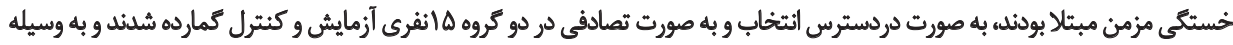

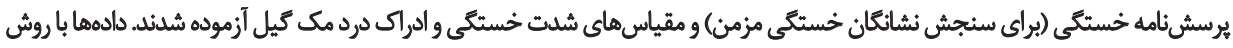

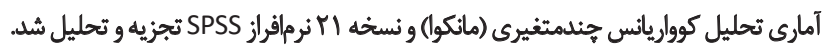

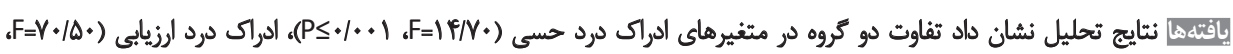

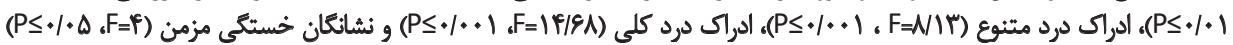

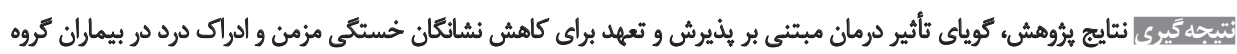

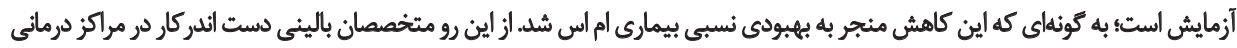

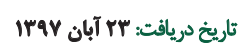

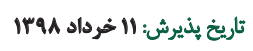

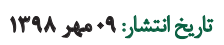

كليدوازوها:

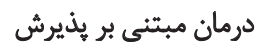

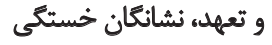
مزمنء ادراك درد، مالتئيل

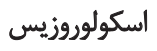

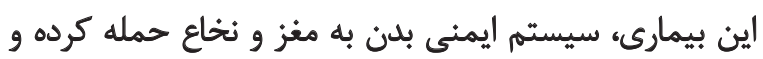

مقدمه

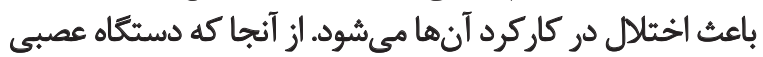

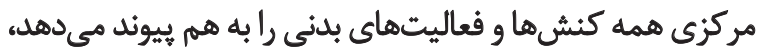

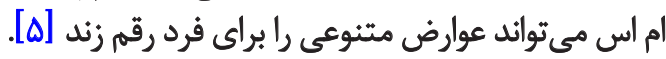

هرجند علت شيوع مشخص نيست، بدون ترديد عوامل

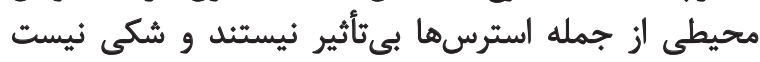

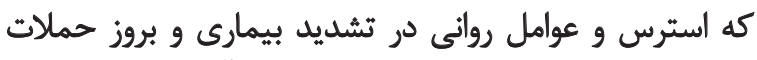

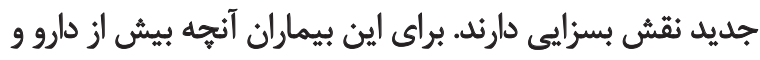

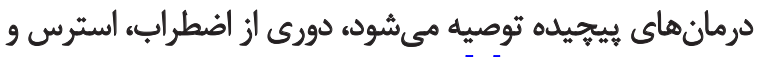

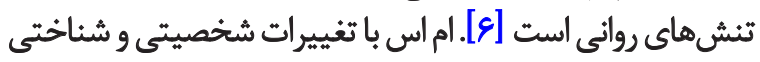

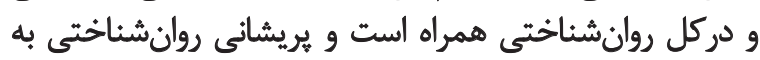

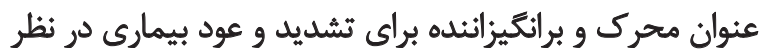

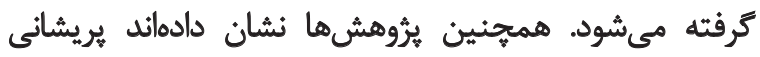

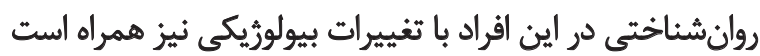

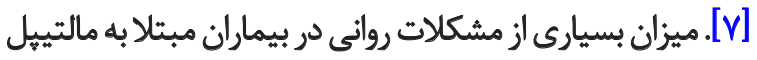

مالتييل اسكولوروزيس' يكى از بيمارىهاى شايع در جوانان

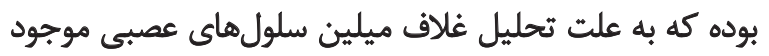

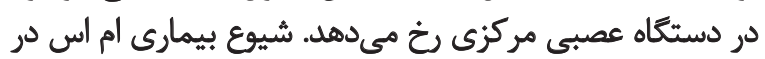

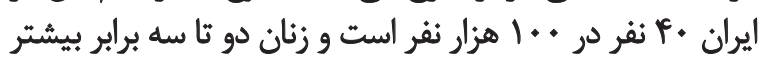

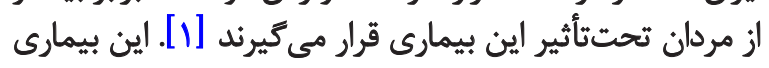

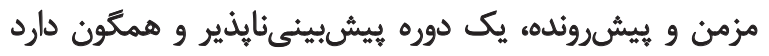

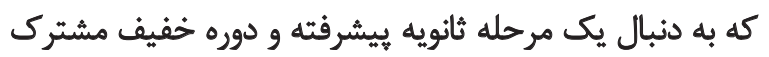

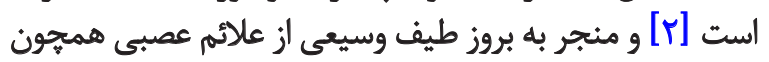

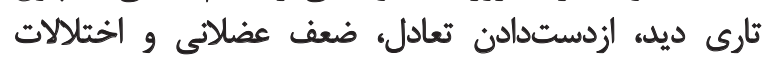

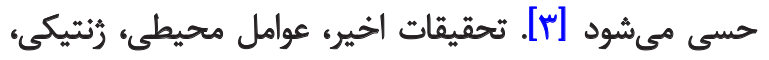

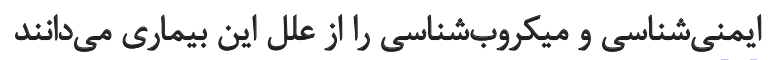

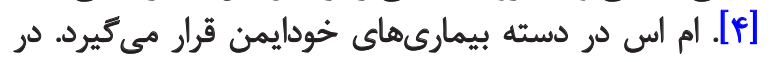

1. Multiple Sclerosis (MS) 


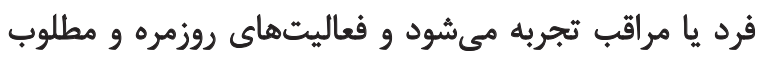

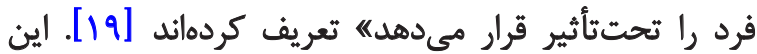

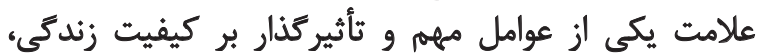

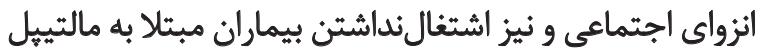

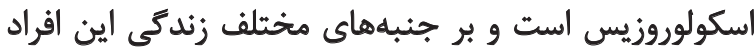

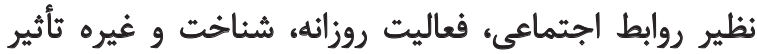

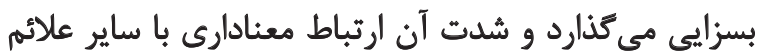

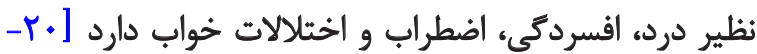

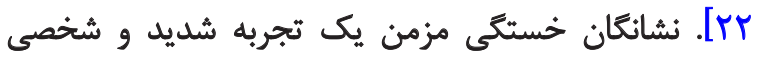

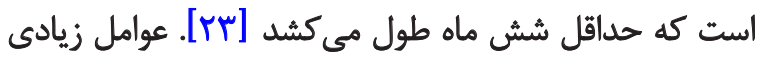

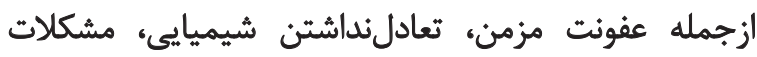

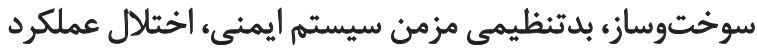

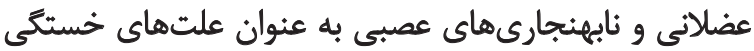

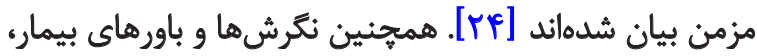

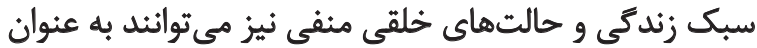
علل روانشناختى مطرح باشند [IV]

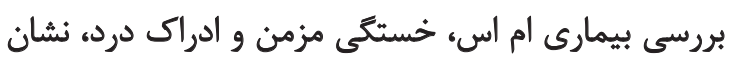

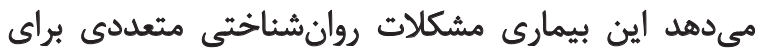

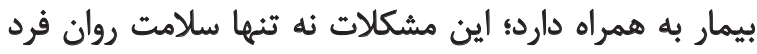

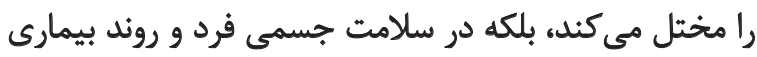

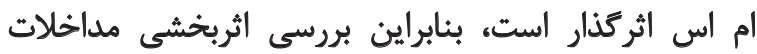

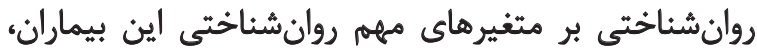

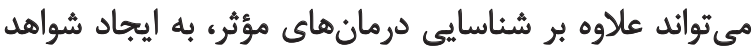

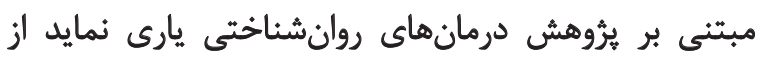

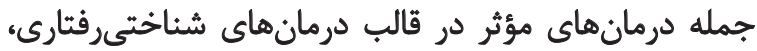

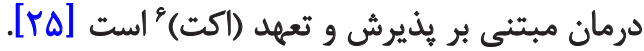

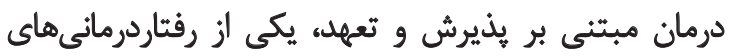

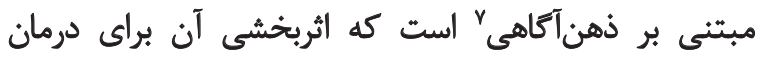

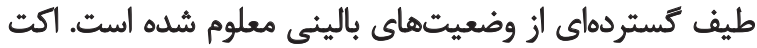
اين فرض را دارد كه فرايندهاى روانشيناختي ذاينى ذهن انسان

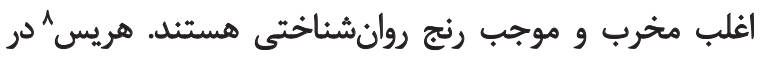

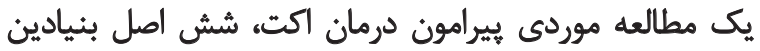

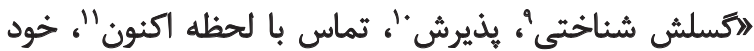

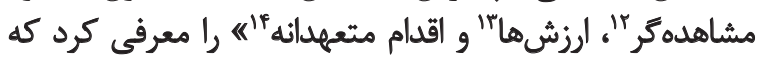

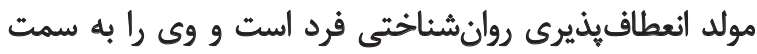

6. Acceptance and Commitment Therapy (ACT)

7. Mindfulness

8. Haris

9. Defusion

10. Acceptance

11. Contact with the present moment

12. Self as context

13. Values

14. Committed action
اسكولوروزيس كزارش شده است [A ، 9] كه بيماران اين مشكلات

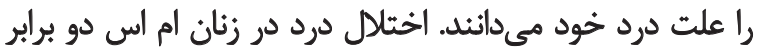

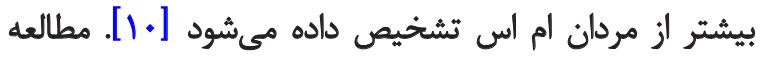

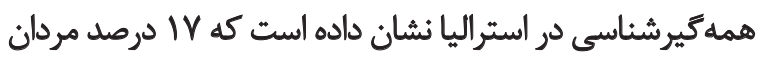

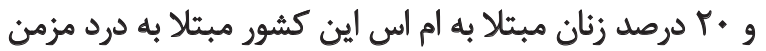

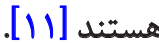

درد احساس ناخوشايندى است كه توسط نورونهاى حسى مايى

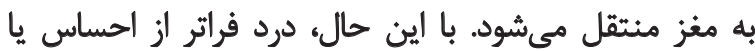

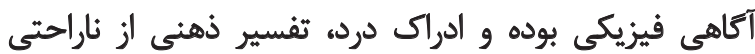

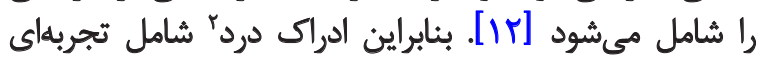

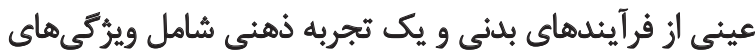

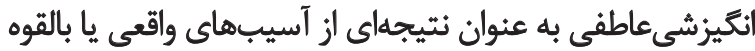

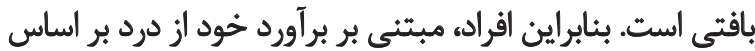

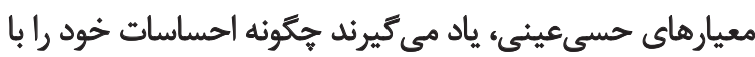

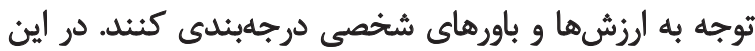

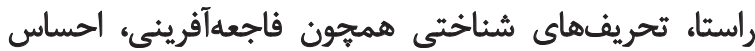

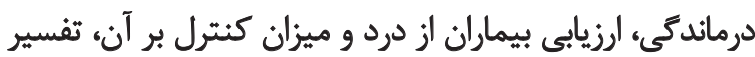

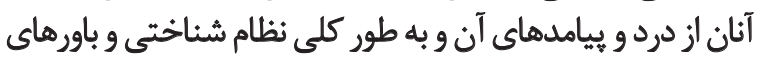

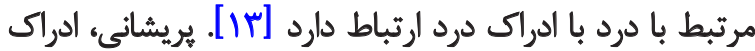

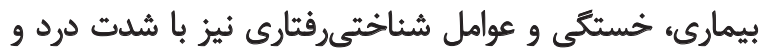

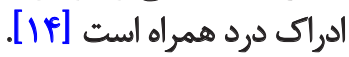

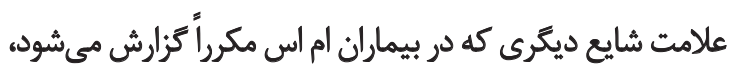

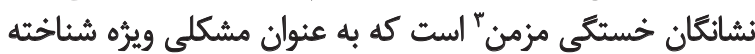

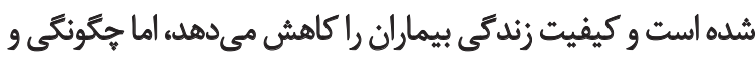

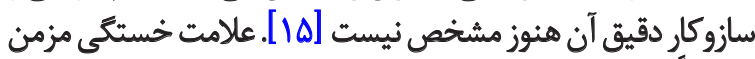

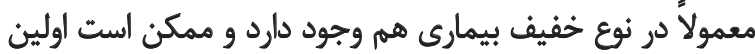

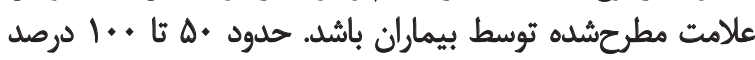

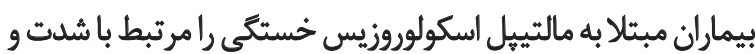

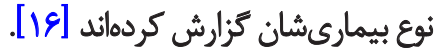

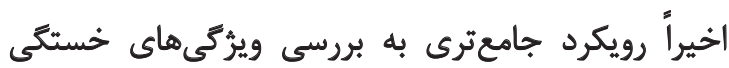

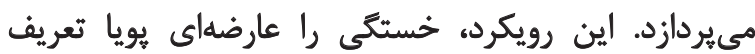

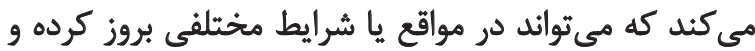

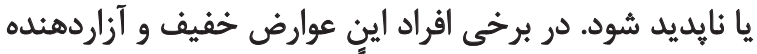

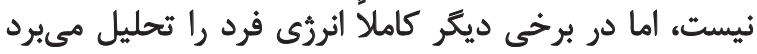

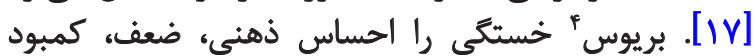

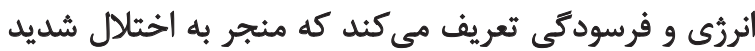

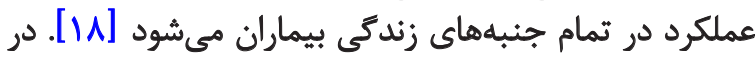

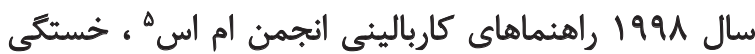

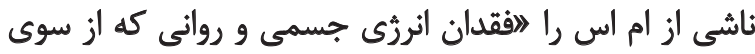

2. Pain Perception

3. Chronic Fatigue Syndrome (CFS)

4. Berrios

5. Multiple sclerosis council of clinical practice 


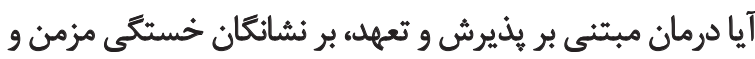

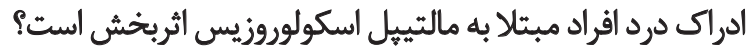

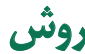

طرح يروهش حاضر كه با كد

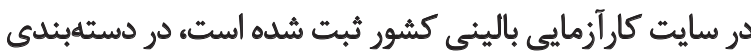

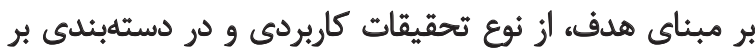

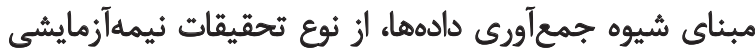

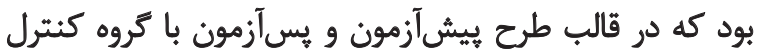

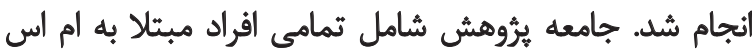

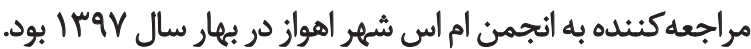

براى انتخاب تروه نمونه واجد شرايط، ابتدا از طريق مراجعه

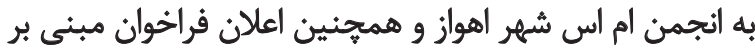

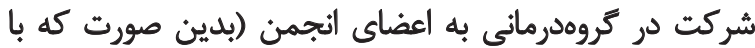

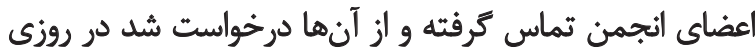

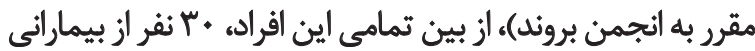

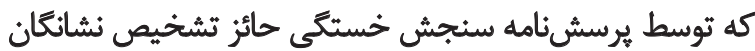

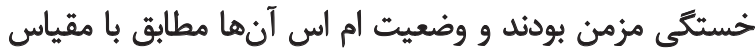

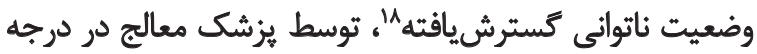

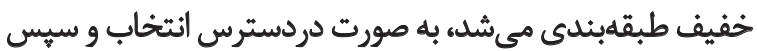

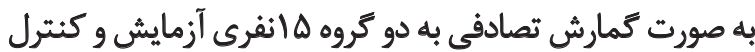

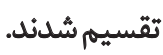

در ثيروهش حاضر، درمان مبتنى بر بذيرش و تعهد متغير

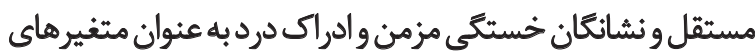

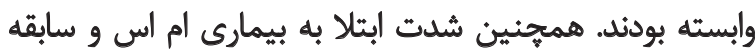

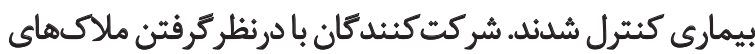

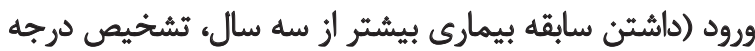

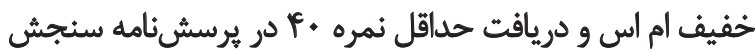

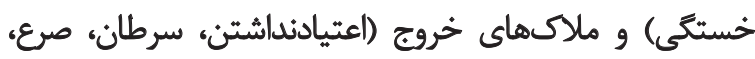

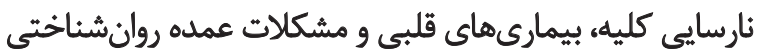

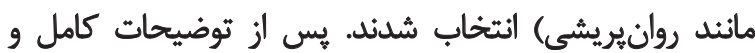

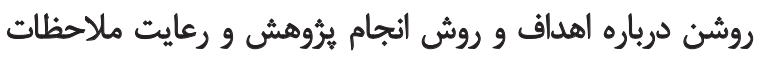

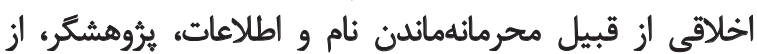

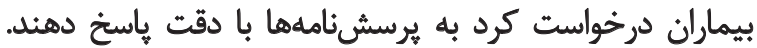

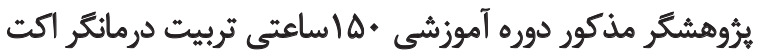

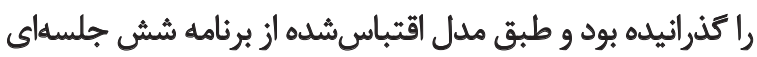

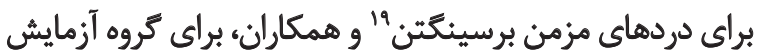

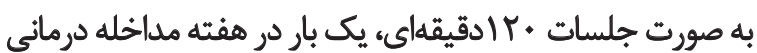

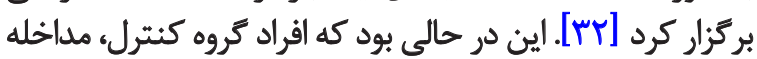

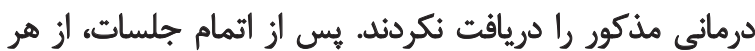

18. Expanded Disability Status Scale (EDSS)

19. Brassington

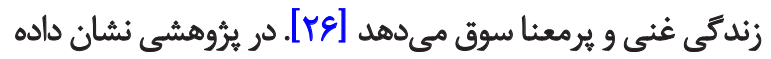

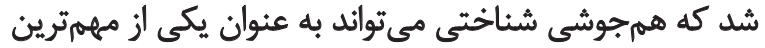

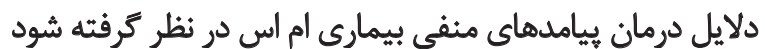

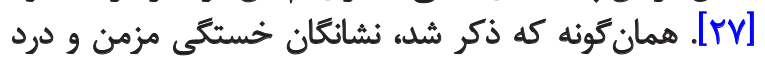
مزمن يكى از علل عمده شكايت در بيماران أم اس است است كند

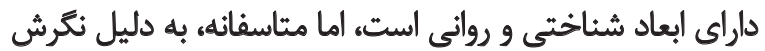

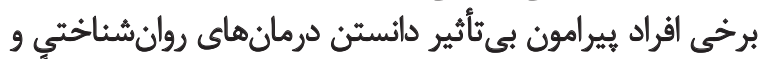

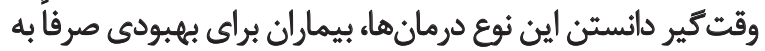
درمان هاى دارويى روى مي آورند.

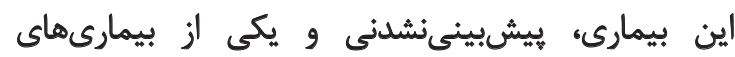

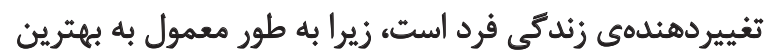

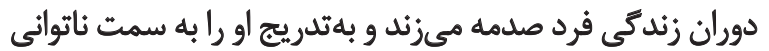

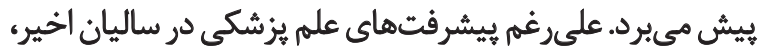

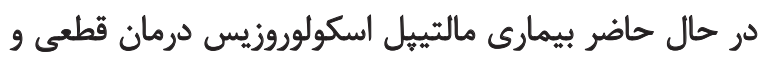

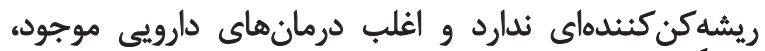

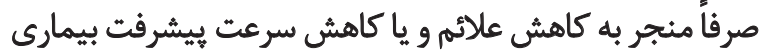

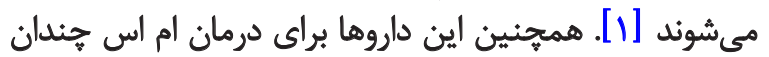

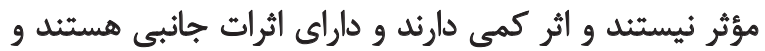

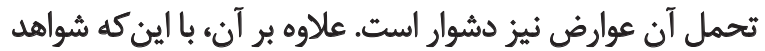

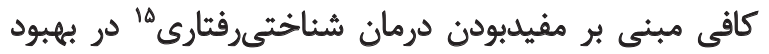

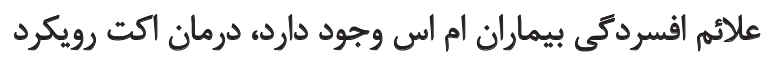

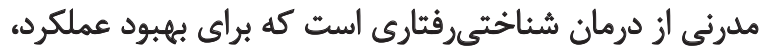

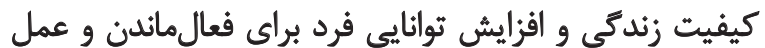

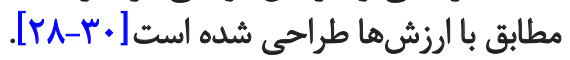
يُؤوهشها همجنين از كارآمدى درمان اكت در مقايسه

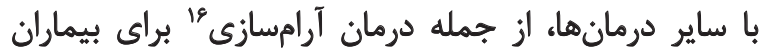

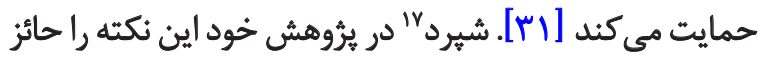

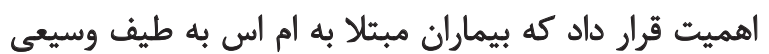

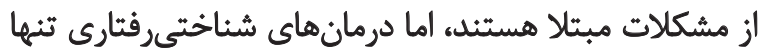

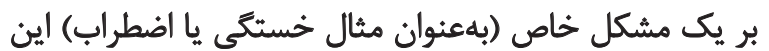

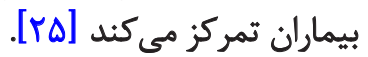

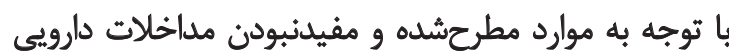

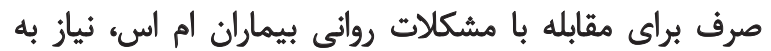

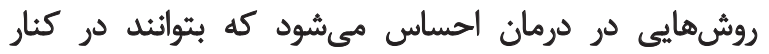
درمانهاى دارويى، علائم بيشترى از بيماري ديمارى (مائند اضطراب،

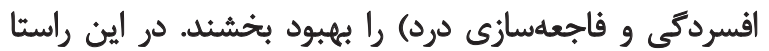

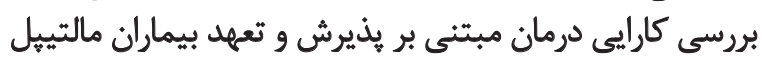

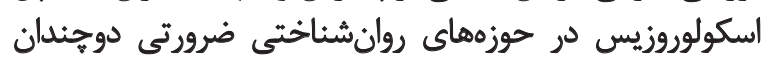

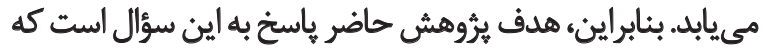

15. Cognitive Behavioural Therapy (CBT)

16. Relaxation Therapy (RT)

17. Sheppard 
جدول ا. خلاصه درمان مبتنى بر هذيرش و تعهيد [بr]

\begin{tabular}{|c|c|c|c|}
\hline تكاليف & محتوا & هدف & شماره جلسه \\
\hline معرفي استعارهها و ارائه تمرين خآنمينى & آشنايى اعضاى كروه با يكديكر و وطرحكردن تسهيل كثندها و منطق & معرفي درمان به كروه & اول \\
\hline همرفى استعاره خركوش سفيد و تمرين & 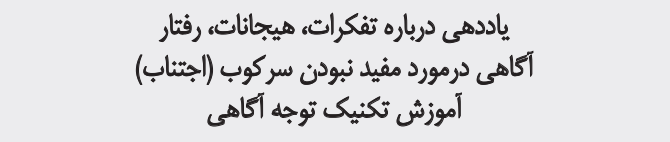 & مدل رفتارى & دوم \\
\hline تمرينات توجه أكاهي و يكيارجهانسازى & فهنم ارزش هاي فردى و شناسايى ارزش ها به اعضا & انتّخاب جهت ارزشها & سوم \\
\hline 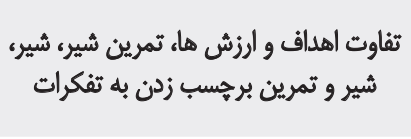 & 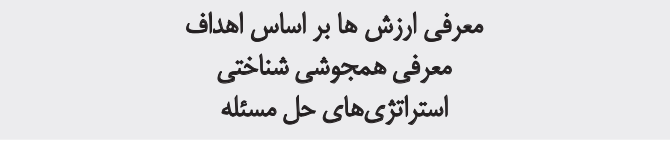 & زندكى با ارزشها & جهارج \\
\hline 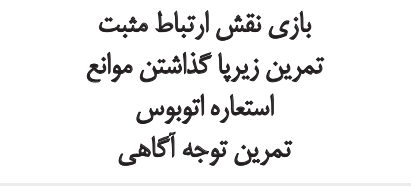 & 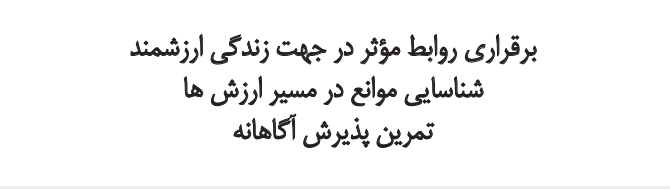 & اقدام ارزشمند & ينجمم \\
\hline 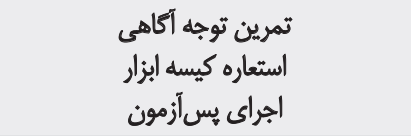 & تجيربه اقراد در خود بانه رابطه و اقدان زمينه متعهد & كار در جهت هايان & ششم: \\
\hline
\end{tabular}

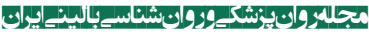

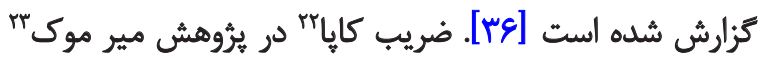

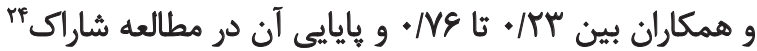

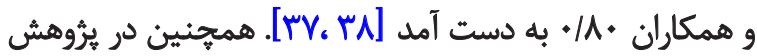

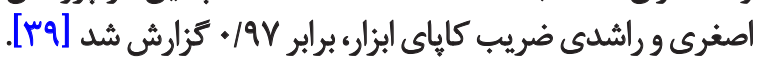

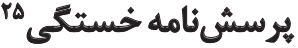

يرسشنامه خستكى براى شناسايى و سنجش نشانكان

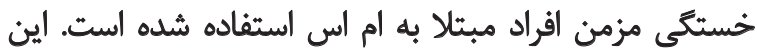

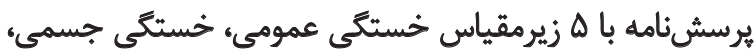

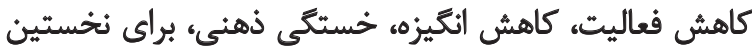

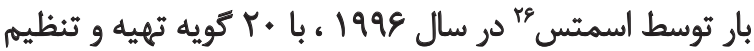

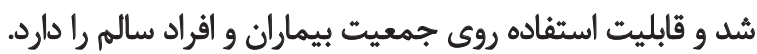

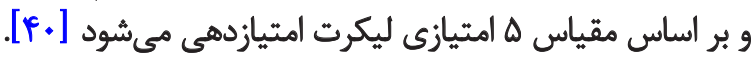

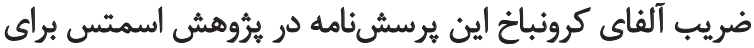

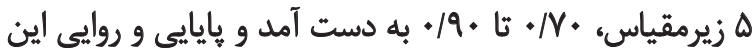

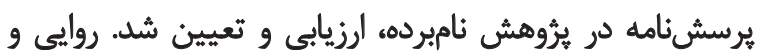

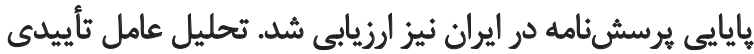

\section{Kappa Coefficient}

23. Meyer-Moock

24. Sharrack

25. Multidimensional Fatigue Inventory (MFI)

26. Smets
دو كروه، يسآزمون به عمل آمد. لازم به ذكر است به بس از اتمام

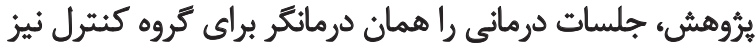

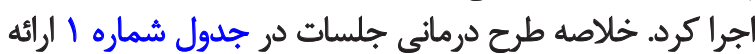

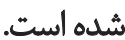

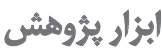

\section{مقياس وضعيت ناتوانى كسترش يافته}

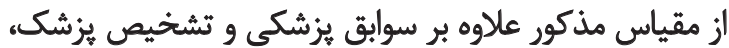

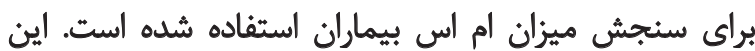

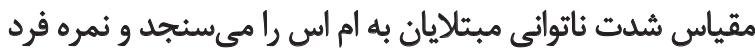

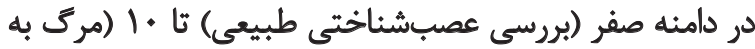

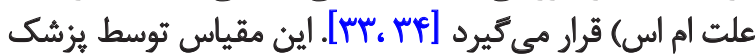

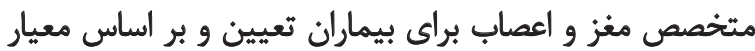

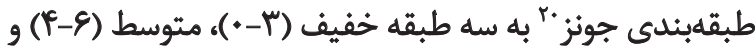

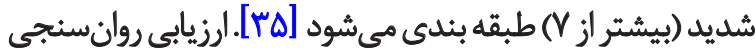

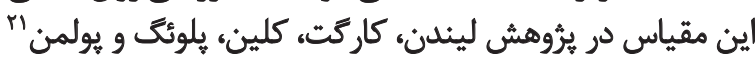

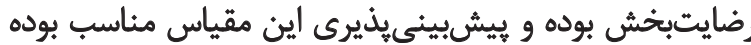

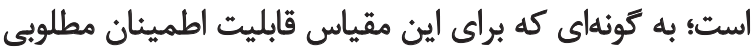

20. Jounz

21. Linden, Kragt, Klein, Ploeg, Polman 
بررسى ادراك درد تهيه و تنظيم كرده است [ـها]. ابزار فوق

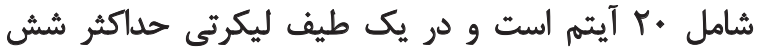

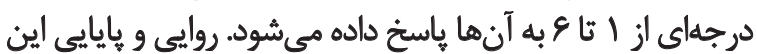

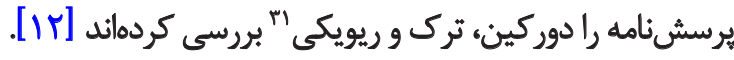

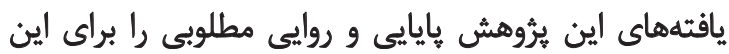

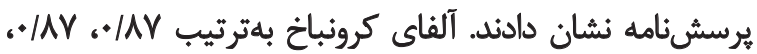

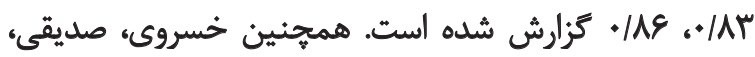

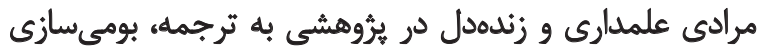

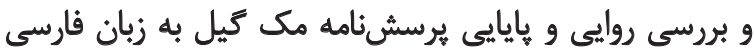

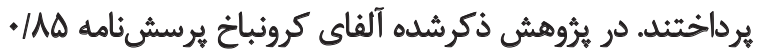

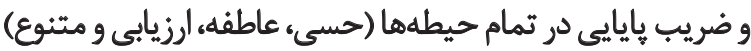

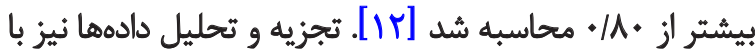

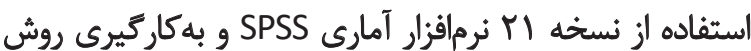
تحليل كوواريانس جندمتغيرى (مانكوا)"آنجام شد.

يافتلها

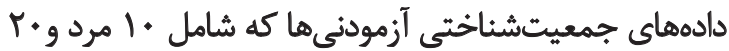

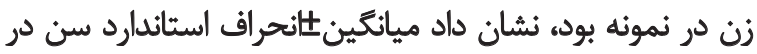

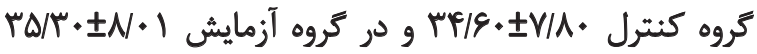

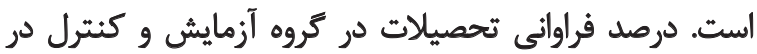

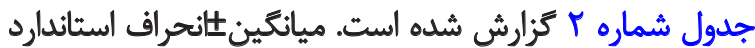

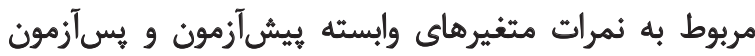

31. Dworkin, Turk \& Revicki

32. Multivariable Analyze of Covariance (MANCOVA)
نشان داد سؤالات هر بعد توصيفكر همان بعد است و يرسشئامه

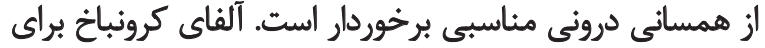

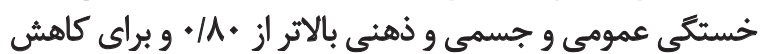

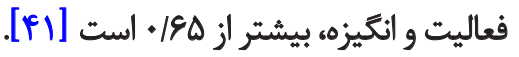

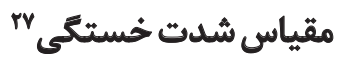

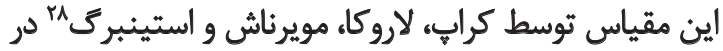

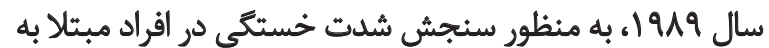

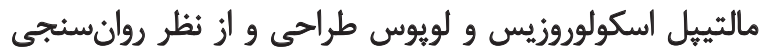

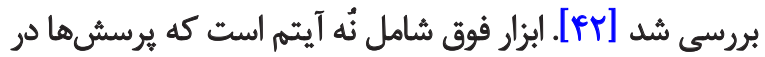

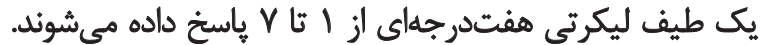

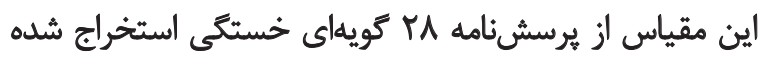

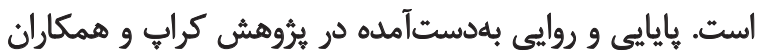

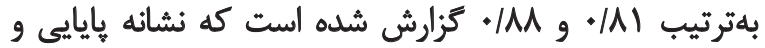

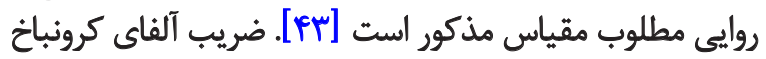

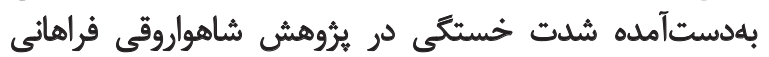

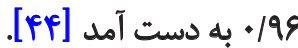

يرسش نامه درد مك كيل

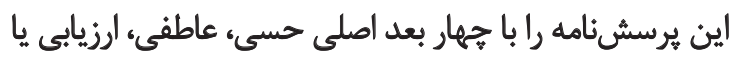

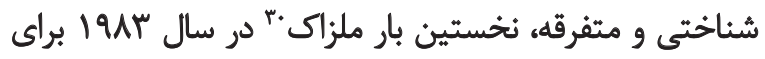

27. Fatigue Severity Scale (FSS)

28. Krupp, LaRocca, Muir-Nash \& Steinberg

29. McGill Pain Questionnaire (MCGPQ)

30. Melzak

جدول r. ويزٔكى هاى جمعيتشناختى نمونه مورد مطالعه از نظر سطوح تحصيلات

\begin{tabular}{|c|c|c|}
\hline فراوائى (درصد) & كروه & تحصيالات \\
\hline$r(r+)$ & أزيمايش & \multirow{2}{*}{ بعىنسواد } \\
\hline$r\left(1 r^{\prime} /{ }^{\prime}\right)$ & كنترل & \\
\hline$r(r+)$ & أزمايش & \multirow{2}{*}{ سيكل } \\
\hline$\cdot(\cdot)$ & كتترل & \\
\hline$r(I r / r)$ & أزمايش & \multirow{2}{*}{ دييلم } \\
\hline$g\left(p^{e}\right)$ & كنترل & \\
\hline$P(Y E / V)$ & آزيهايش & \multirow{2}{*}{ فوق دييلم } \\
\hline$r(r \cdot)$ & كنترل & \\
\hline$r(I r / T)$ & آزمايش & \multirow{2}{*}{ ليسائس } \\
\hline$r(I r / T)$ & كترل & \\
\hline $1(E / V)$ & أزمايش & \multirow{2}{*}{ فوق ليسائس } \\
\hline$\cdot(\cdot)$ & كنترل & \\
\hline
\end{tabular}


جدول ". ميانكين و انحراف معيار نمرات بيش آزمون و يس آزمون به تفكيك كروهها

\begin{tabular}{|c|c|c|c|}
\hline \multicolumn{2}{|c|}{ مياتكين بانحراف معيار } & \multicolumn{2}{|c|}{ مثير هاى بُؤشش } \\
\hline IV/ITEV/AY & $\mathrm{r} / / \mathrm{r} \pm \mathrm{q} / \mathrm{q}$. & آزمايش & \multirow{2}{*}{ ادراى درد حسى } \\
\hline$|V / r \cdot \pm V / V|$ & $11 / \Delta r \pm s / q \varepsilon$ & كنترل & \\
\hline$\Delta / r+ \pm r / q V$ & $\Delta / r+ \pm r / g V$ & آزمايش & \multirow{2}{*}{ ادراك درد عاطفى } \\
\hline$F / Y r \pm r / \Delta F$ & $F / M T+Y / \Delta F$ & كتترل & \\
\hline$I / M T \pm I / M$ & $Y / \bullet \pm \pm / A Y$ & آزآمايش & \multirow{2}{*}{ ادراك درد الرزيابي } \\
\hline$\cdot / A Y \pm \cdot / M$ & $\cdot / 8 \cdot \pm \cdot / N$ & كتشرل & \\
\hline$V / M^{\sim} \pm 8 / m$ & V/AY $\pm \& /$ & آزمايش & \multirow{2}{*}{ ادراى درد متنوع } \\
\hline$\varepsilon / 8 . \pm r / T q$ & $F / A V \pm F / / \Delta$ & كتثل & \\
\hline$\pi / \% \cdot \pm 1 / \mu$ & $r W / q \Psi \pm Y / . r$ & أزمايش & \multirow{2}{*}{ ادراى درد كل } \\
\hline$r q / e+ \pm 1 / r^{2}$. & $r / N \Psi \pm 1 / T r$ & كنترل & \\
\hline$r \Delta / N V^{\top} \pm V / A V$ & $M V / r) \pm V / \Delta \Delta$ & آزمايش & \multirow{2}{*}{ شانكان خستكى مزمن } \\
\hline$P r / . V \pm V / . q$ & $m / r \cdot \pm V / \cdot r$ & كنترل & \\
\hline
\end{tabular}

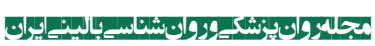

جدول F.نتايج تحليل كوواريانس جندمتغيره يكراهه روى ميانكين نمرهماى بس آزمون با كنترل بيشآزمون

\begin{tabular}{|c|c|c|c|c|c|c|}
\hline اندازه اثٔر & $p$ & $f$ & مجذنور ميائكَين & df & مجموع مجذورات & مثغير وابسته \\
\hline.$/ 98$ & $.1 .+1$ & $10 / 10$ & $1 \cdot A T / M I$ & 1 & $1 \cdot A T / M$ & ادراك درد كل \\
\hline$. / \Delta \mid$ & .1 .9 & F/TV & reg/rV & 1 & res/rV & نشانكان خُستى مزمن \\
\hline
\end{tabular}

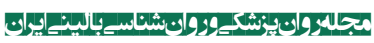

تحليل كوواريانس جندمتغيرى در اين بثروهش بلامانع است. بر اساس آزمون مائكوا، نتايج بهدست آمده براى لامبداي

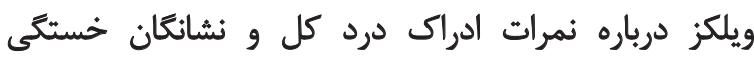

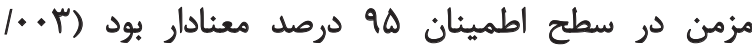

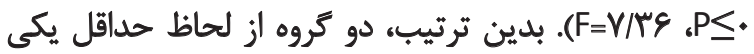

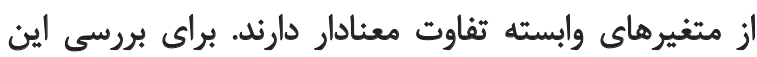

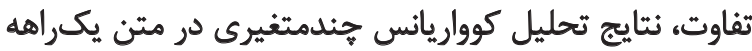

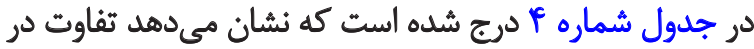

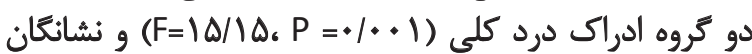

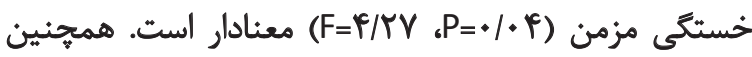

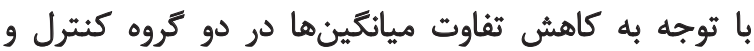

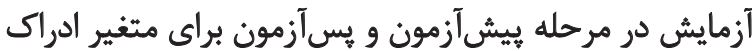

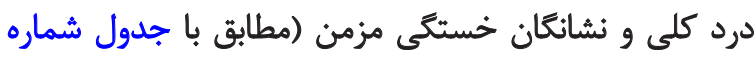

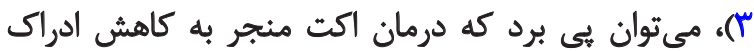

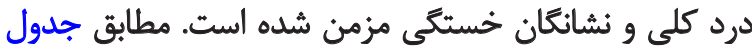

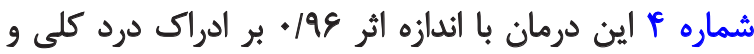

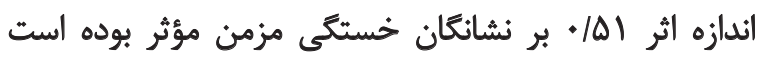

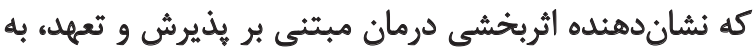

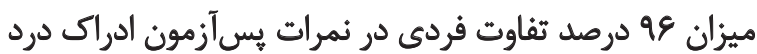

ثرؤش در دو كروه كنترل و آزمايش نيز در جدول شماره r درج شده است. نتايج بررسى ويشفرض هاي كوض

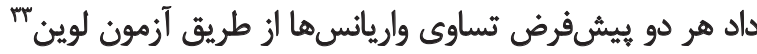

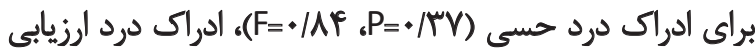

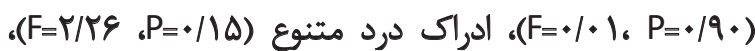

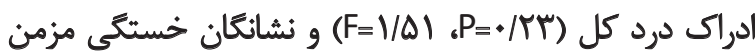

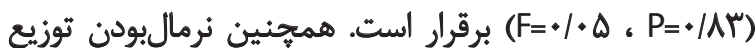

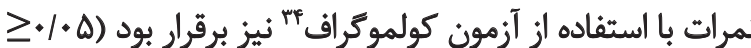

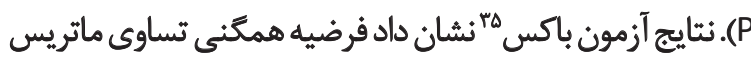

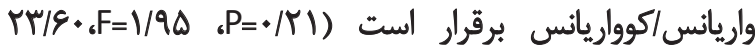
BoX=

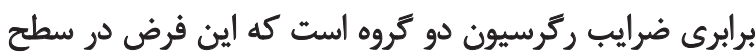

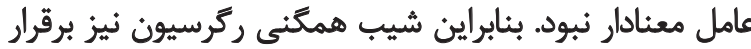

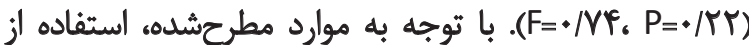

\section{Levenes test}

34. Kolmogorov- Smirnov test

35. Box's M test (BOX) 
جدوله. نتايج تحليل كوواريائس جند متغيره يكراهه روى ميانكين نمرههاى يس آزمون با كنترل بيش آزمون

\begin{tabular}{|c|c|c|c|c|c|c|}
\hline اندازه اثي & $\mathbf{p}$ & $f$ & مجذور ميانكين & df & مجموع مجذورات & متغير وابسته \\
\hline.$/ 4$ & $+1+.1$ & IVIIP & $P \Delta \Delta / \cdot T$ & 1 & $r \Delta Q /+r$ & ادراك درد حسى \\
\hline.$/ 11$ & $+1+1$ & $r / r$. & $11 / 9 r$ & 1 & $11 / 94$ & ادراى درد عاطفى \\
\hline$\cdot / M$ &..++4 & $1 . / \Delta F$ & V/D. & 1 & $9 / \Delta$. & ادراك درد ارزيابى \\
\hline - /AF & $+1+\infty$ & $9 / \Delta 1$ & $1+\Delta / H F$ & 1 & $1+\Delta / \pi F$ & ادراك درد متنوع \\
\hline
\end{tabular}

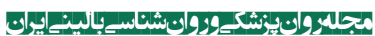

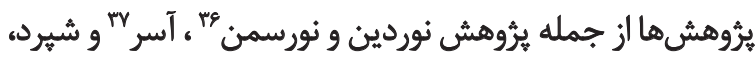

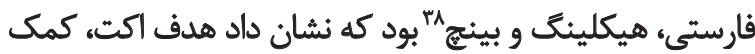

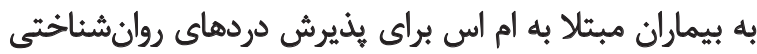

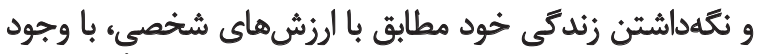

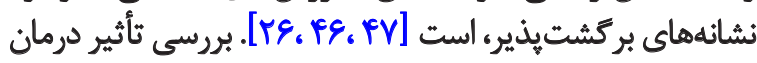

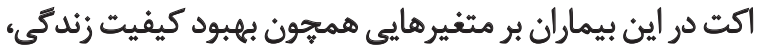

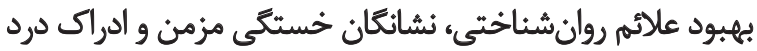

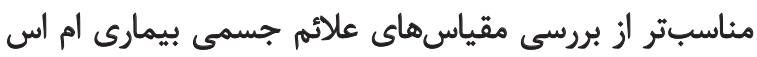

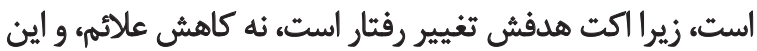

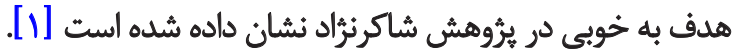

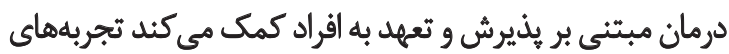

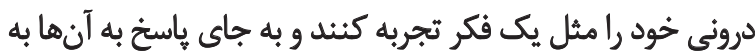

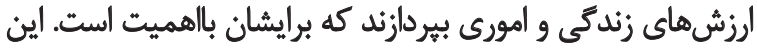

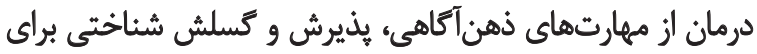

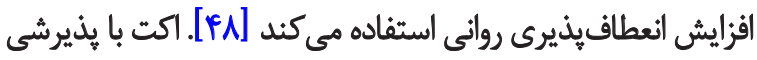

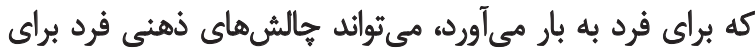

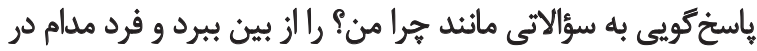

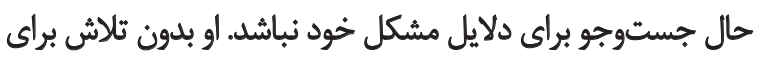

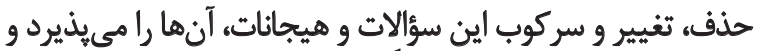

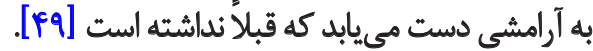

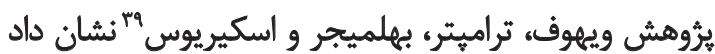

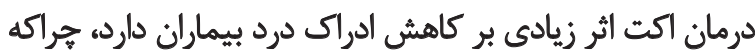

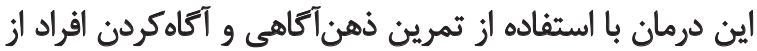

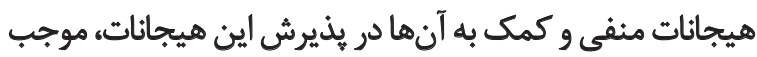

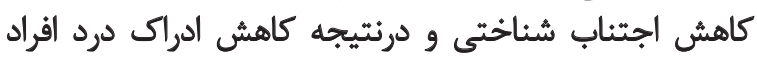

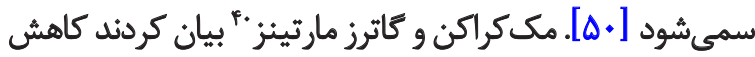

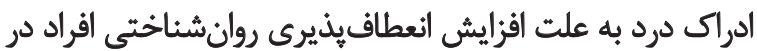

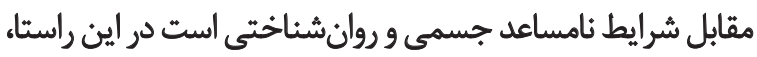

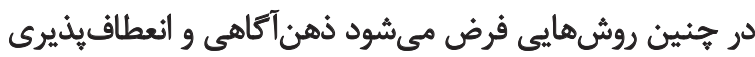

\section{Nordin \& Rorsman}

37. Oser

38. Sheppard, Forsyth, Hickling \& Bianchi

39. Veehof, Tromptter, Bohlmeijer \& Scheruse

40. McCracken, Gutiérrez-Martínez
كلى و اه درصد تفاوت فردى در نمرات يسآزمون نشانكان خستخى مزمن است.

لامبداى ويلكز بهدست آمده براى خرده مقياسهاى ادراى دردي

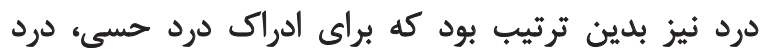

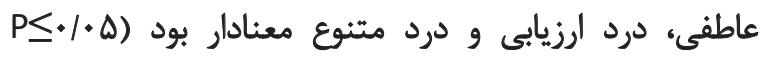
تفاوت SF/lV،

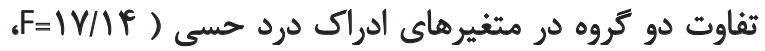

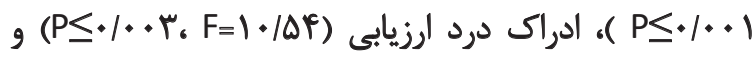

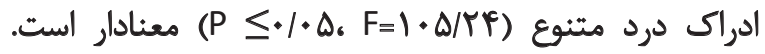

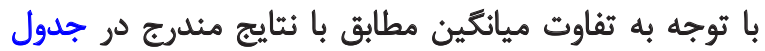

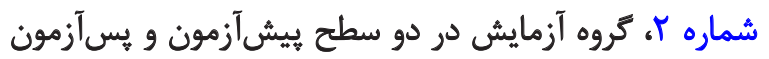

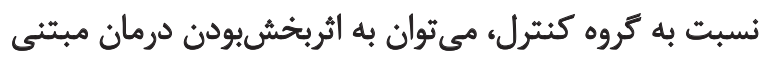

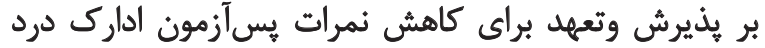

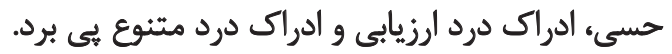

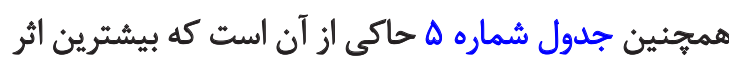

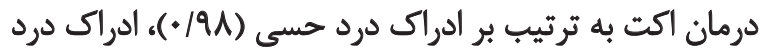

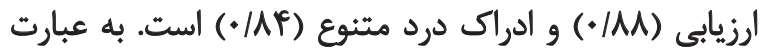

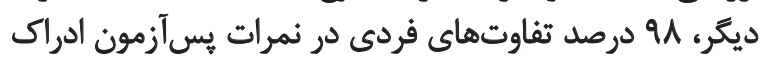

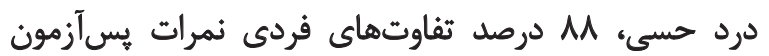

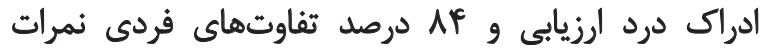

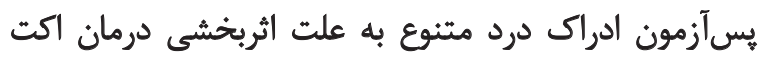

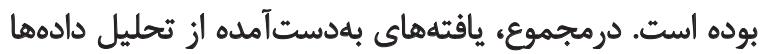

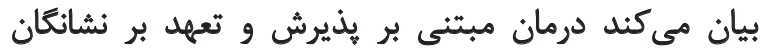

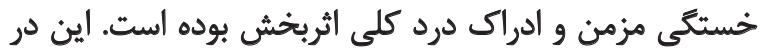

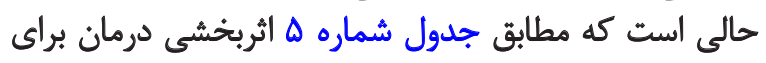
ادراك درد عاطفى معنادار نبوده است

بحث

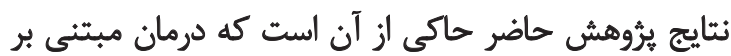

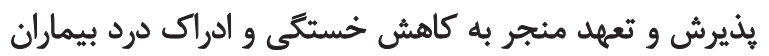

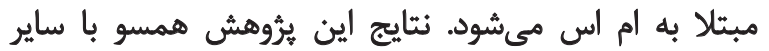


اجتماعى كمترى دارند و نسبت به اقراد سالم با سطوح بسيار

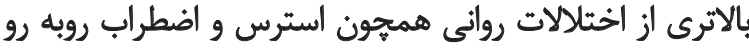

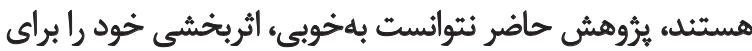

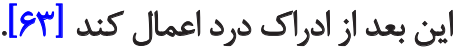

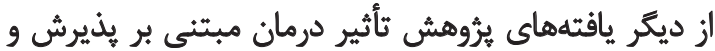

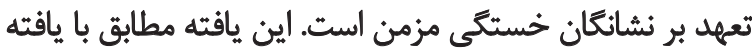

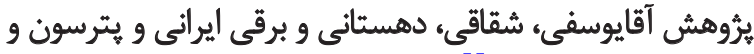

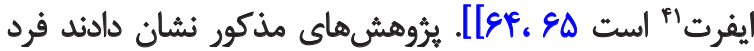

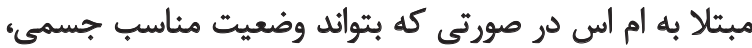

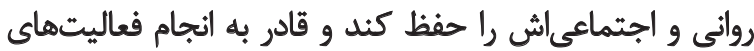

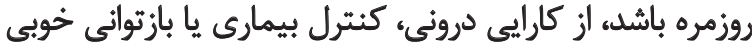

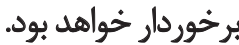
اكت با اقزايش ثأثير مثبت كرايى، خوشبينى بر ادراك بيمارى

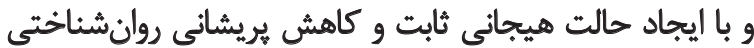

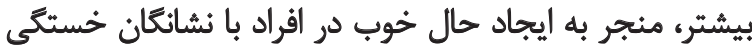

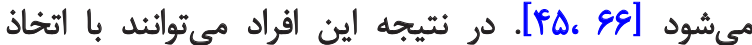

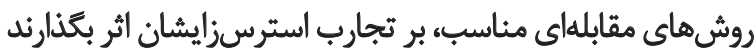

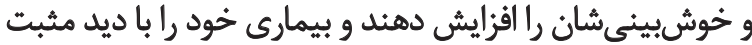

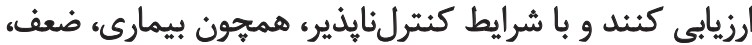

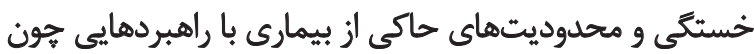

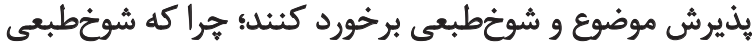

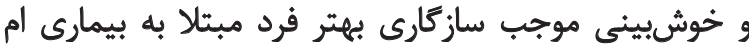

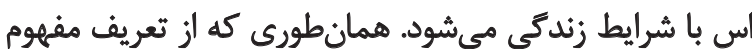

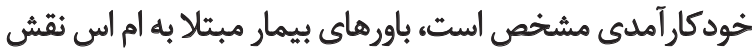

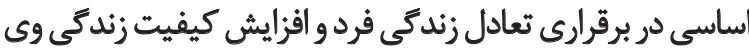

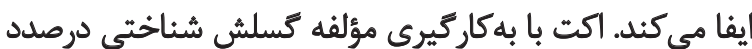

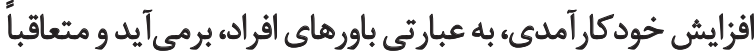

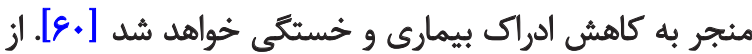
اينرو يافته مذكور نيز دور از انتظار نبوده است.

\section{نتيجليرى}

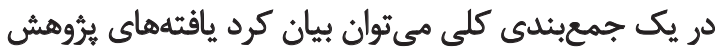

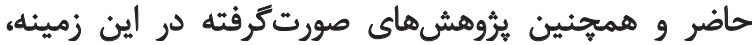

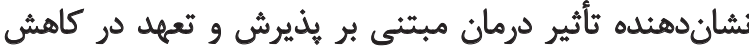

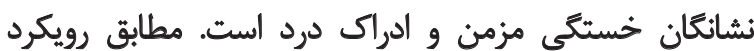

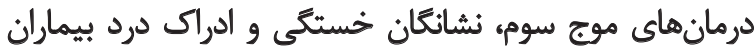

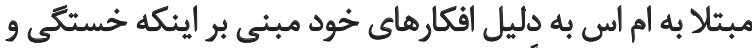

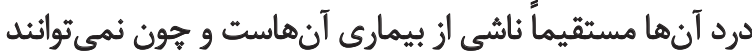

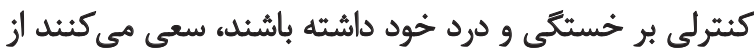

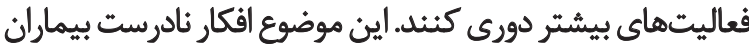

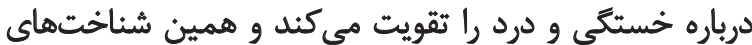

41. Peterson \& Eifert

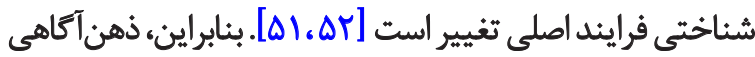

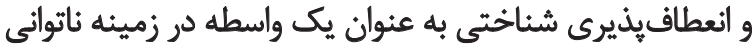

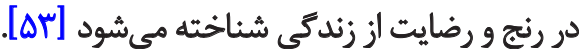

اكت با مطرحكردن مؤلفه بذيرش و كسلش به دنبال

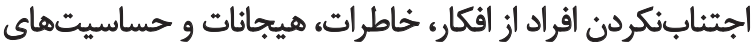

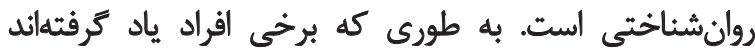

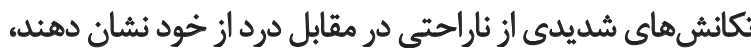

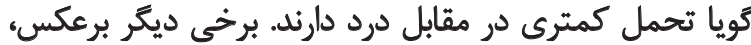

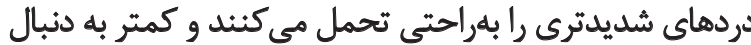

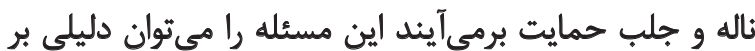

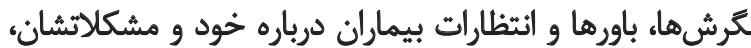

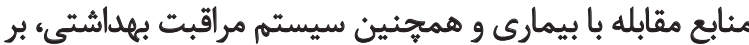

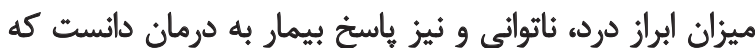

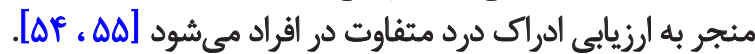
اكت با آموزش درباره تميزدادن بين مسائل و توصيف آنها

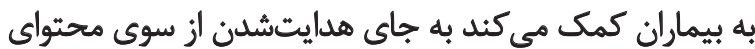

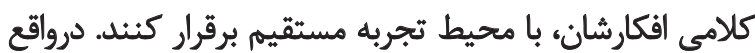

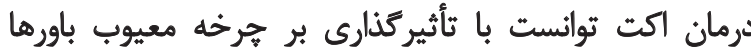

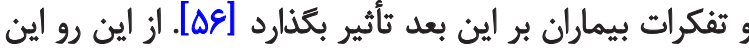

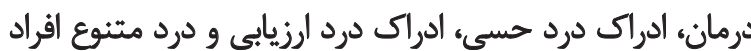

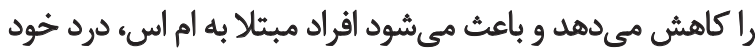

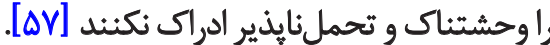

درمان اكت باعث ميشود حتى اكر تجربه واقعى درد بدون

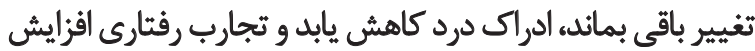

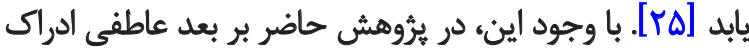

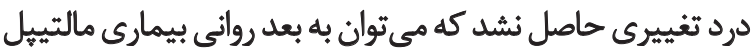

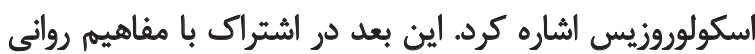

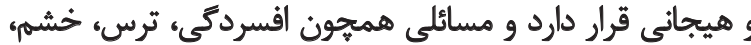

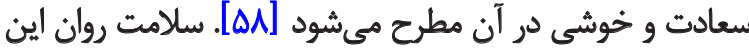

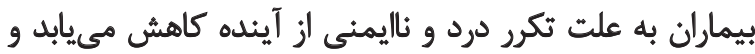

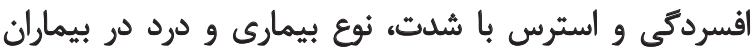

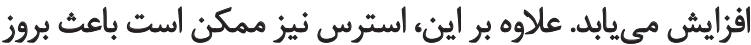

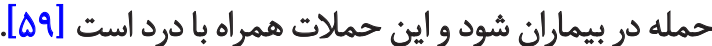
به طور كلى هر فرد در زمان و شرايط خاص، آستانه تحمل

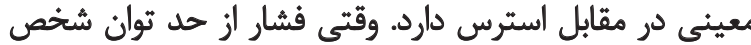

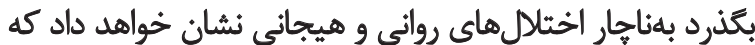

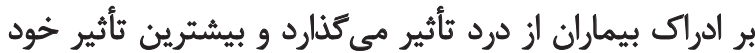

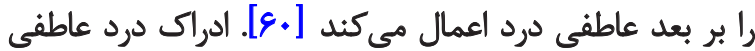

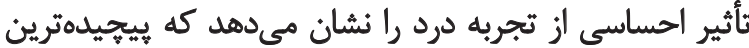

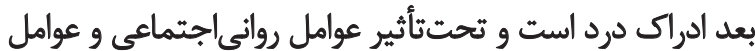

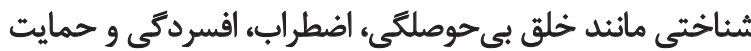

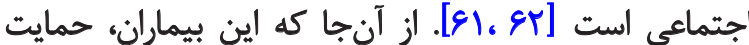


سياسيكرًارى

از كليه برسنل انجمن ام اس شهرستان اهواز بهويزوه خانم

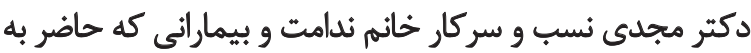

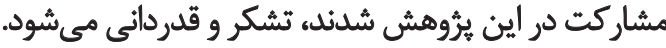

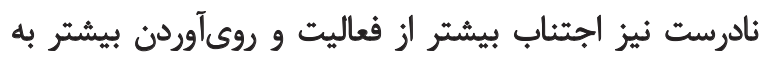

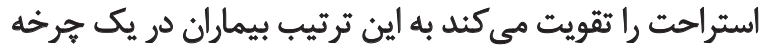

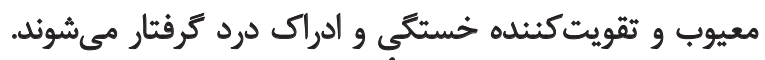

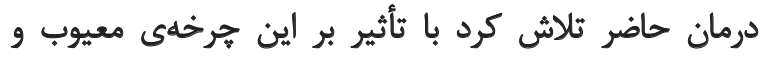

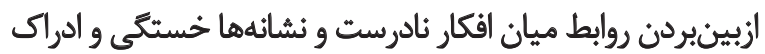

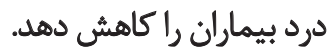

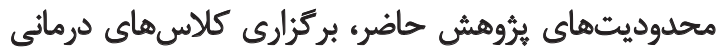

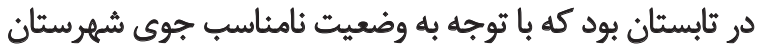

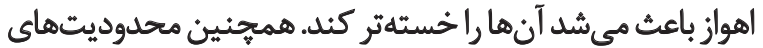

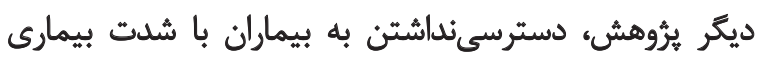

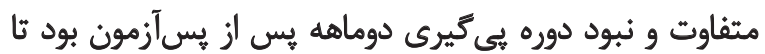
بررسي تأثير كذارى درمان دقيقتر اعمال شود.

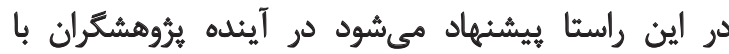

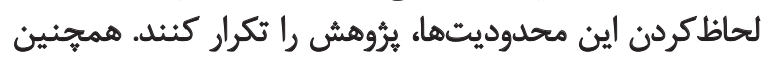

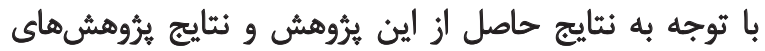

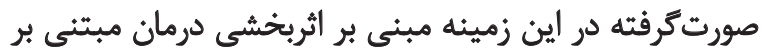

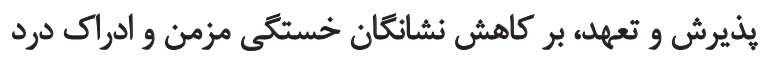

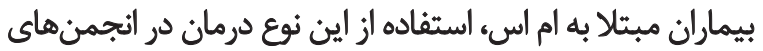

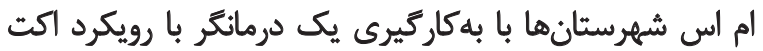
بيشنهادمى مشود.

ملاحظات اخلاقى

\section{ييروى أزاصول اخلاق ئوهش}

در اين تحقيق ، فرم ملاحظات اخلاقى توسط بيماران تكميل

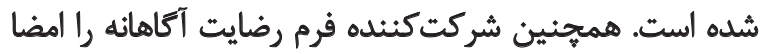
كردند و حق داشتند كه هر زمان خواستند مطالعه راترك كنيند.

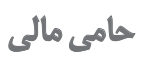

يُروهش حاضر بركرفته از ياياننامه كارشناسىارشد نويسنده

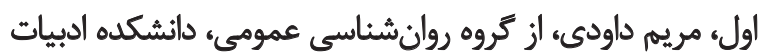

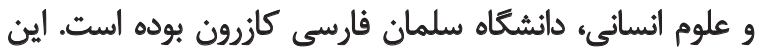
ئروهش، حامى مالى نداشته است.

مشاركت ثويسند مكان

مفهوم سازى، اعتبار سنجي و مديريت يرورةه: همه نويسندكان؛

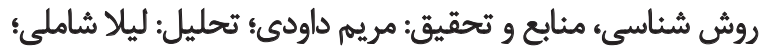
نكارش يُشنويس: مريم داودي؛ ويرايش و نهايیىسازي: ليلائ

$$
\text { ت تعارض مناقع }
$$

بنا به اظهار نويسندكان، اين مقاله تعارض منافع ندارد. 


\section{References}

[1] Shakernezhad S, Moaze N, Hamidi M, Hashemi R, Barazande $\mathrm{N}$, Badaghi, M. [The effectiveness of adequate treatment and commitment to psychological parasitics, marital satisfaction and quality of life in women with Multiple Sclerosis (Persian)]. Journal of Health Care. 2017; 1(19):7-17.

[2] Reynders T, D'haeseleer M, De Keyser J, Nagels GB, D'hooghe $\mathrm{M}$. Definition, prevalence and predictive factors of benign multiple sclerosis. Neurological Science. 2017; 5(7):37-43. [DOI:10. 1016/j. ensci. 2017. 05. 002] [PMID] [PMCID]

[3] Zahoor I, Haq E. Multiple Sclerosis in India: Iceberg or volcano. Journal of Neuroimmunology. 2017; 6(307):27-30. [DOI:10. 1016/j. jneuroim. 2017. 03. 015] [PMID]

[4] Cimenti H, Bornem S, Nikki T, Frederike H, Larissa K, Andreas S. Neuraxial anesthesia in patients with multiple sclerosis: A systematic review. Revista Brasileira de Anestesiologia. 2017; 67(4):404-10. [DOI:10. 1016/j. bjane. 2016. 09. 001]

[5] Lee EY. Application of myelin water imaging to detect diffuse white matter damage in multiple sclerosis. [MSc. thesis]. British Columbia: University of British Columbia; 2019. [DOI: 10. 14288/1. 0376036]

[6] Mirhashemi M, Najafi F. [The effectiveness of solution therapy on resiliency and sense of coherence in Multiple Sclerosis (MS) patients (Persion)]. Medical Journal of the Islamic Republic of Iran. 2014; 24 (3):175-81.

[7] Mitsonis CI, Potagas C, Zervas I, Sfagos K. The effects of stressful life events on the course of multiple sclerosis: A review. International Journal of Neuroscience. 2009; 119(3):315-35. [DOI:10. 1080/00207450802480192] [PMID]

[8] Archibald CJ, McGrath PJ, Ritvo PG, Fisk JD, Bhan V, Maxner $\mathrm{CE}$, et al. Pain prevalence, severity and impact in a clinic sample of multiple sclerosis patients. Pain. 1994; 58(1):89-93. [DOI:10. 1016/0304-3959(94)90188-0]

[9] Jensen MP, Chodroff MJ, Dworkin RH. The impact of neuropathic pain on health-related quality of life: Review and implications. Neurology. 2007; 68(15):1178-82. [DOI:10. 1212/01. wnl. 0000259085. 61898. 9e] [PMID]

[10] Mirzamani Bafghi SM, Sadiidi A, Safari A. [Comparison of the effect of pain on various aspects of life in the elderly and those with chronic pain (Persion)]. Salmand: Iranian Journal of Aging. 2018; 3(7):47-55

[11] Blyth FM, March LM, Brnabic AJM, Jorm LR, Williamso M, Cousins MJ. Chronic pain in Australia: A prevalence study. Pain 2001; 89(2-3):127-34. [DOI:10. 1016/S0304-3959(00)00355-9]

[12] Dworkin RH, Turk DC, Revicki DA, Harding G, Coyne KS, Peirce-Sandner S, et al. Development and initial validation of an expanded and revised version of the Short-form McGill Pain Questionnaire (SF-MPQ-2). Pain. 2009; 144(1):35-42. [DOI:10. 1016/j. pain. 2009. 02. 007] [PMID]

[13] Turk DC, Gatchel RJ. Psychological approaches to pain management: A practitioner's handbook. New York: Guilford Press; 2006.

[14] Chisari C, Chilcot J. The experience of pain severity and pain interference in vulvodynia patients: The role of cognitive-behavioural factors, psychological distress and fatigue. Journal of Psy- chosomatic Research. 2017; 93:83-9. [DOI:10. 1016/j. jpsychores. 2016. 12. 010] [PMID]

[15] Akcali A, Zengin F, Nuraksoy S, Zengin O. Fatigue in multiple sclerosis: Is it related to cytokines and hypothalamic-pituitaryadrenal axis? Multiple Sclerosis and Related Disorders. 2017 7(15):37-41. [DOI:10. 1016/j. msard. 2017. 03. 004] [PMID]

[16] Pittion-Vouyovitch SDM, Guillemin F, Vandenberghe N. Anxionnat R, Vespignani H. Fatigue in Multiple Sclerosis is related to disability, depression and quality of life. Journal of the Neurological Sciences. 2006; 243(12):39-45. [DOI:10. 1016/j. jns. 2005. 11. 025] [PMID]

[17] Ennis M, Webster S. Living with fatigue: Fatigue management for people with MS. Letchworth Garden: Multiple Sclerosis Trust Spirella Building; 2015.

[18] Berrios GE. Feeling of fatigue and psychopathology: A conceptual history. Psychiatry. 1990; 31(2): 140-151. [DOI:10. 1016/0010-440X(90)90018-N]

[19] Multiple Sclerosis Council of Clinical Practice Guidelines. Fatigue and multiple sclerosis: Evidence-based management strategies for fatigue in multiple sclerosis. Washington DC: Paralyzed Veterans of America; 1998.

[20] Motahari Nejad F, Parvaneh S, Ghahari S. [Fatigue in patients with multiple sclerosis: Causes, evaluation and treatment (Persion)]. Journal of Paramedical Sciences and Rehabilitation. 2016; 5(1):73-80.

[21] Osborne TL, Turner AP, Williams RM, Bowen JD, Hatzakis $\mathrm{M}$, Rodriguez A, et al. Correlates of pain interference in multiple sclerosis. Rehabilitation Psychology. 2006; 51(2):166-74. [DOI:10. 1037/0090-5550.51. 2. 166]

[22] Attarian HP, Brown KM, Duntley SP, Carter JD, Cross AH. The relationship of sleep disturbances and fatigue in multiple sclerosis. Archives of Neurology. 2004; 61(4):525-8. [DOI:10. 1001/archneur. 61. 4. 525] [PMID]

[23] Fukuda K, Strauss SE, Hickie I, Sharpe MC, Dobbins JG Komaroff A. The chronic fatigue syndrome: A comprehensive approach to its definition and study. Journal of Chronic Fatigue Syndrome. 1995; 1(2):67-84. [DOI:10. 7326/0003-4819-121-12199412150-00009] [PMID]

[24] ] Sajjadi A, Abotalebi Gh. [Chronic fatigue, from diagnosis to relief (Persion)]. AJA University of Medical Sciences. 2012; 12(1): $18-22$

[25] Sheppard SC, Forsyth JP, Hicklin EJ, Bianchi JM. A novel application of acceptance and commitment therapy for psychosocial problems associated with multiple sclerosis: Results from a halfday workshop intervention. International Journal of MS Care. 2010; 12 (4):200-6. [DOI:10. 7224/1537-2073-12. 4. 200]

[26] Harris R. Theoretical Basis Treatment Based on Commitment and Admission in Simple Language (Part II). [A Feizi, Persian trans.] Tehran: The Psychological Services and Life Advice Center website; 2014

[27] Valvano A, Floyd RM, Penwell-Waines L, Stepleman L, Lewis $\mathrm{K}$, House A. The relationship between cognitive fusion, stigma and well-being in people with multiple sclerosis. Journal of Contextual Behavioral Science. 2016; 5(4):266-70. [DOI:10.1016/j. jcbs. 2016. 07. 003]

[28] Kappos L, Radue EW, Comi G, Montalban X, Butzkueven H Wiendl $\mathrm{H}$, et al. Switching fromnatalizumab to fingolimod: A 
randomized, placebocontrolled study in RRMS. Neurology. 2015; 85(1):29-39. [DOI:10. 1212/WNL. 0000000000001706] [PMID] [PMCID]

[29] Thomas PW, Thomas S, Hillier C, Galvin K, Baker R. Psychological interventions for multiple sclerosis. Cochrane Database of Systematic Reviews. 2006; 1(25): CD004431. [DOI:10. 1002/14651858. CD004431. pub2]

[30] Hayes SC, Strosahl K, Wilson KG. Acceptance and commitment therapy: $\mathrm{N}$ experiential approach to behavior change. New York: Guilford; 1999.

[31] Twohig MP, Hayes SC, Plumb JC, Pruitt LD, Collins AB, Hazlett- Stevens $\mathrm{H}$, et al. A randomized clinical trial of acceptance and commitment therapy versus progressive relaxation training for obsessive compulsive disorder. Journal of Consulting and Clinical Psychology. 2010; 78(5):705-76. [DOI:10. 1037/a0020508] [PMID] [PMCID]

[32] Brassington L, Ferreira NB, Yates S, Fearn J, Lanza P, Kemp K, Gillanders D. Better living with illness: A transdiagnostic acceptance and commitment therapy group intervention for chronic physical illness. Journal of Contextual Behavioral Science. 2016; 5(4):208-14. [DOI:10. 1016/j. jcbs. 2016. 09. 001]

[33] Ashjazadeh N, Hadianfard H, Feridoni S, Farjam E. Assessment of health-related quality of life in patients with multiple sclerosis living in the Fars province of Iran. Neurol Neuroimmunol Neuroinflamm. 2016; 3(3):57-62. [DOI:10. 20517/2347-8659. 2015. 40]

[34] Kurtzke JF. Rating neurologic impairment in multiple sclerosis: An Expanded Disability Status Scale (EDSS). Neurology. 1983; 11(33):1444-52. [DOI:10. 1212/WNL. 33. 11. 1444] [PMID]

[35] Fletcher SG, Castro-Borrero W, Remington G, Treadaway K, Lemack GE, Frohman EM. Sexual dysfunction in patients with multiple sclerosis: A multidisciplinary approach to evaluation and man-agement. Nature Clinical Practice Urology. 2009; 6(2):96-107. [DOI:10. 1038/ncpuro1298] [PMID]

[36] Linden F, Kragt JJ, Klei M, Ploeg H, Polman CH. Psychometric evalutain of the Multiple Sclerosis Impact Scale (MSIS-29) for proxy use. Journal of Neurology, Neurosurgery, and Psychiatry. 2005; 76(12):1677-981. [DOI:10. 1136/jnnp. 2005. 065227] [PMID] [PMCID]

[37] Meyer-Moock S, Feng YS, Maeurer M, Dippel FW, Kohlmann T. Systematic literature review and validity evaluation of the Expanded Disability Status Scale (EDSS) and the Multiple Sclerosis Functional Composite (MSFC) in patients with multiple sclerosis. BMC Neurology, 2014; (14)58, 1-10. [DOI: 10. 1186/1471-2377-1458]

[38] Sharrack B, Hughes RAC, Soudain S, Dunn G. The psychometric properties of clinical ratingscales used in Multiple Sclerosis. Brain. 1999; 122(1):141-59. [DOI: 10. 1093/brain/122. 1. 141]

[39] Asghari E, Rashedi I. [Study of disability (based on EDSS) and other variables in 100 patients with clinically definite multiple sclerosis referring to hospitals of Tehran Medical Sciences University and private centers (Persian)]. [MSc. thesis]. Tehran: Tehran Medical Sciences University; 1999.

[40] Smets EM, Garssen B, Cull A, De Haes JC. Application of the Multidimensional Fatigue Inventory (MFI-20) in cancer patients receiving radiotherapy. British Journal of Cancer. 1996; 73(2):2415. [DOI:10. 1038/bjc. 1996. 42] [PMID] [PMCID]

[41] Najafi Mehri S, Pashandi S, Mahmoodi H, Ebadi A, Ghanei M. [Assessment of fatigue and spirometery parameters in chemical war victims with respiratory disease. Iran (Persion)]. Iranian Journal of Public Health. 2010; 2(4):29-35.

[42] Krupp LB, LaRocca NG, Muir-Nash J, Steinberg AD. The fatigue severity scale: Application to patients with multiple sclerosis and systemic lupus erythematosus. Archives of Neurology. 1989; 46(10):1121-3. [DOI:10. 1001/archneur. 1989. 00520460115022] [PMID]

[43] Shahvaraghani A, Azimian M, Falahpoor M, Karimlo M. [Evaluation of reliability and validity of the Persian version of Fatigue Severity Scale (FSS) among persons with multiple sclerosis (Pesion)]. Archives of Rehabilitation. 2014; 13(4):84-91.

[44] Melzack R. Development and initial validation of an expanded and revised version of the Short-form McGill Pain Questionnaire (SF-MPQ-2). Pain. 2009; 144(1):35-42. [DOI:10. 1016/j. pain. 2009. 02. 007] [PMID]

[45] Nordin L, Rorsman IA. Cognitive behaiviaral therapy in Multiple Scolerosis: A randomaized controlled pilot study of acceptance and commitment therapy. Journal of Rehabilitation Medicine. 2012; 44(1):87-90. [DOI:10. 2340/16501977-0898] [PMID]

[46] Oser M. Acceptance and Commitment Therapy for Multiple Sclerosis. Boston: Harvard Medical School; 2015.

[47] Forman EM, Herbert JD. New directions in cognitive behavior therapy: Acceptance-based therapies. In: O'Donohue WT, Fisher JE, editors. General Principles and Empirically Supported Techniques of Cognitive Behavior Therapy. Hoboken: John Wiley \& Sons; 2009.

[48] Hamill TS, Pickett SM, Amsbaugh HM, Aho KM. Mindfulness and acceptance in relation to Behavioral Inhibition System sensitivity and psychological distress. Personality and Individual Differences. 2015; 72(1):24-9. [DOI:10. 1016/j. paid. 2014. 08. 007]

[49] Veehof MM, Tromptter HR, Bohlmeijer KM, Scheruse KMG. Acceptance- and mindfulness-based interventions for the treatment of chronic pain: A meta-analytic review. Cognitive Behaviour Therapy. 2015; 45(1):5-31. [DOI:10. 1080/16506073. 2015. 1098724] [PMID]

[50] Mirzahosseini H, Poorabdol S, Sobhi Gharamaki N, Sarvani S. [The effect of acceptance and commitment therapy in decreasing cognitive avoidance among students with Specific Learning Disorder (SLD) (Persion)]. Iranian Journal of Psychiatry \& Clinical Psychology. 2018; 22(3):166-75. [DOI:10. 18869/acadpub. ijpcp. 22. 3.166]

[51] McCracken LM, Gutiérrez-Martínez O. Processes of change in psychological flexibility in an interdisciplinary group-based treatment for chronic pain based on acceptance and commitment therapy. Behaviour Research and Therapy. 2011; 49(4):267-74. [DOI:10. 1016/j. brat. 2011. 02. 004] [PMID]

[52] Rajabi Gh, Yousefali A. [Psychology of pain, etiology, evaluation and treatment (Persion)]. Psychotherapical Novelties. 1998; 7(25-26):70-95.

[53] McCracken LM, Morley S. The psychological flexibility model: A basis for integration and progress in psychological approaches to chronic pain management. The Journal of Pain. 2014; 15(3):22134. [DOI:10. 1016/j.jpain. 2013. 10. 014] [PMID]

[54] Albert U, Mania G, Bogetto F, Chiorle A, Matain-Cols D. Clinical predictors of health-related quality of life. Psychiatry. 2015; 51(2):193-200. [DOI:10. 1016/j. comppsych. 2009. 03. 004] [PMID] 
[55] Amiri S, Eisazadegan A, Alilu M, Banafsheh, M. [Study of pain experience, social support and perceptual beliefs in people with chronic pain (Persion)]. Journal of Neyshabur University of Medical Sciences. 2016; 4(2): 58-68.

[56] Bach P, Moran DJ. [ACT in action: Conceptualization in treatment based on admission and commitment (Persian)]. Tehran: Arjmand Publications; 2014.

[57] Turk DC, Melzack R. Handbook of pain assessment. New York: Guilford Press; 2010.

[58] Sadock BJ, Sadock VA, Ruiz P. Kaplan and Sadock comperehensive Text book of psychiatry. Philadelphia: Lippincott Williams \& Wilkins; 2012.

[59] Alavi MS, Jabal Ameli S. [The effectiveness of cognitive-behavioral therapy on emotional control of MS patients in the City of Isfahan (Persian)]. Jorjani Biomedicine Journal. 2018; 6(1):44-54. [DOI:10.18869/acadpub. hrjbaq. 1.4. 215]

[60] Amir F, Ahadi H, Nikkhah K, Seirafi M. [The effectiveness of acceptance and commitment group therapy and group logotherapy in reducing perceived stress among MS patients (Persian)]. Caspian Journal of Neurological Sciences. 2017; 3(11):175-84.

[61] Mason ST, Arceneaux LL, Abouhassan W, Lauterbach D, Seebach C, Fauerbach JA. Confirmatory factor analysis of the Short Form McGill Pain Questionnaire with burn patients. Eplasty. 2008; 9(8):1-5. [PMID] [PMCID]

[62] Hakimjavadi M, Lavasani MGH, Haghighatgi M, Zebardast O. [Relationship among depression, anxiety, stress and personality in veteran children (Persian)]. Iranian Journal of War and Public Health. 2013; 3(1):9-16.

[63] Sheydayi Aghdam Sh, Shamsuddini Lori S, Abbasi S, Yousefi S, Abdollahi S, Moradi J. [The effectiveness of treatment based on acceptance and commitment in reducing distress and inefficient attitudes in patients with MS (Persion)]. Journal of Clinical Psychology Andishe va Raftar. 2014; 9(34):57-88

[64] Agayyoshifi A, Shaghaki F, Mehestani M, Irani Z. [The relationship between Quality of Life (QOL) and psychological capital with illness perception in MS patients (Persion]. Quarterly Journal of the Royal Meteorological Society. 2012; 1(1): 32-45.

[65] Peterson BD, Eifert GH. Using acceptance and commitment therapy to treat infertility stress. Cognitive and Behavioral Practice. 2011; 18(4):577-87. [DOI:10. 1016/j. cbpra. 2010. 03. 004]

[66] Van Damme S, Crombez G, Van Houdenhove B, Mariman A, Michielsen W. Well-being in patients with chronic fatigue syndrome: The role of acceptance. Journal of Psychosomatic Research. 2006; 61(5):595-9. [DOI:10. 1016/j. jpsychores. 2006. 04. 015] [PMID] 
This Page Intentionally Left Blank 